\title{
Neurophysiological and Behavioral Effects of Anti-Orexinergic Treatments in a Mouse Model of Huntington's Disease
}

\author{
Magali Cabanas ${ }^{1,2} \cdot$ Cristiana Pistono $^{1,2} \cdot$ Laura Puygrenier $^{1,2} \cdot$ Divyangana Rakesh $^{1,2} \cdot$ Yannick Jeantet $^{1,2}$. \\ Maurice Garret $^{1,2} \cdot$ Yoon H. Cho ${ }^{1,2}$
}

Published online: 26 March 2019

(C) The American Society for Experimental NeuroTherapeutics, Inc. 2019

\begin{abstract}
Huntington's disease (HD) is associated with sleep and circadian disturbances in addition to hallmark motor and cognitive impairments. Electrophysiological studies on HD mouse models have revealed an aberrant oscillatory activity at the beta frequency, during sleep, that is associated with HD pathology. Moreover, HD animal models display an abnormal sleep-wake cycle and sleep fragmentation. In this study, we investigated a potential involvement of the orexinergic system dysfunctioning in sleep-wake and circadian disturbances and abnormal network (i.e., beta) activity in the R6/1 mouse model. We found that the age at which orexin activity starts to deviate from normal activity pattern coincides with that of sleep disturbances as well as the beta activity. We also found that acute administration of Suvorexant, an orexin 1 and orexin 2 receptor antagonist, was sufficient to decrease the beta power significantly and to improve sleep in R6/1 mice. In addition, a 5-day treatment paradigm alleviated cognitive deficits and induced a gain of body weight in female HD mice. These results suggest that restoring normal activity of the orexinergic system could be an efficient therapeutic solution for sleep and behavioral disturbances in HD.
\end{abstract}

Keywords Orexin 1 and 2 receptor antagonist $\cdot$ Sleep $\cdot$ Beta activity $\cdot$ Cognitive deficits $\cdot$ R6/1 mice

\section{Introduction}

Huntington's disease (HD) is a hereditary neurodegenerative disease caused by the mutation of the gene that encodes for the huntingtin protein. The mutation induces an abnormally expanded repetition of trinucleotide CAG encoding for glutamine [1]. In HD, striatal neurons undergo early selective degeneration, then the cellular pathology manifests throughout the brain and periphery at later stages of the disease [2]. Cognitive and neuropsychiatric disturbances are often expressed early and before the appearance of the wellknown symptoms of chorea and other motor deficits. In addition, sleep alterations are present at motor presymptomatic stages of the disease and are characterized by an increase in

Yoon H. Cho

yoon.cho@u-bordeaux.fr

1 Institute of Cognitive and Integrative Neuroscience of Aquitaine, CNRS UMR 5287, Allee Geoffroy St Hilaire, CS 50023, 33615 Pessac Cedex, France

2 Institute of Cognitive and Integrative Neuroscience of Aquitaine, University of Bordeaux, Bordeaux, France daytime sleepiness, which significantly affects a patient's quality of life [3-6].

Numerous HD mouse models also develop early and significant sleep disturbances [7-13]. At the behavioral level, HD mice show disturbances in the sleep-wake architecture leading to sleep fragmentation and increased sleepiness during their active period. In the R6/1 model, sleep abnormalities appear as early as 2 months of age, whereas other behavioral deficits, such as cognitive impairments, are mostly still absent $[10,14-16]$. Motor and cognitive disturbances arise at 3 months of age in this model. Several studies have demonstrated that improving sleep or imposing a regular sleep-wake cycle is beneficial for alleviating cognitive impairments in several other HD mouse models [17-24].

In addition, electroencephalographic (EEG) recordings in several lines of HD mice have highlighted an excessive synchrony of neural activity in the frequencies between 20 and $40 \mathrm{~Hz}$, which is absent in WT littermates [8, 9, 12, 14, 25]. This aberrant oscillatory activity, which is associated with HD, and which we have termed "beta activity" (low gamma activity by other authors), reaches, in R6/1 mice, its maximal intensity during the cortical desynchronized brain state of rapid eye movement (REM) sleep. The beta activity is attenuated 
during slow wave sleep (SWS) and waking in R6/1 mice [14], while it is equally prevalent across these stages in R6/2 mice and Q175 mice $[8,9,12]$. In addition, the phenomenon increases its intensity with age in all models of HD, even though the age of beta appearance differs from each other.

The timing of sleep and wakefulness is affected by changes in the activity of the orexin/hypocretin system. The orexin (Orx) neuropeptide is synthesized by neurons located in the lateral hypothalamic area (LHA) [26], which is a brain region involved in the sleep-wake cycle and other vital behaviors, such as foodseeking and thermoregulation. The Orx neurons in this area promote wakefulness by activating arousal brain areas during the active period $[27,28]$. On the contrary, melanin concentrating hormone $(\mathrm{MCH})$-releasing neurons, also located in the LHA [29], project to the same targets as Orx neurons and inhibit activity of arousal centers during sleep [30,31]. The importance of Orx neurons for consolidating the normal sleep-wake cycle is highlighted by the fact that Orx knockout (KO) mice [27] serve as animal models for narcolepsy.

A loss of Orx neurons has been observed in the postmortem brains of HD patients [32, 33], despite Orx levels in the cerebrospinal fluid being relatively normal [34-36]. Moreover, R6/2 transgenic mice display an unusual Orx activity pattern with respect to their activity-inactivity period, as revealed by c-Fos immunostaining studies [37]. More precisely, Orx activity in the mutant mice is significantly higher during the inactive (light period for rodents), compared to WT littermates. On the contrary, during the active (dark) period, R6/2 mice show a decreased Orx activity, compared to WT animals.

We therefore investigated if the Orx system may also be dysfunctional in R6/1 mice at different ages and stages of disease development using the same c-Fos imaging technique. We found increased Orx activity during the inactive period as in R6/2 mice [37]. Therefore, we verified whether the abnormal increase could be associated with the generation of beta activity and sleep disturbance. Because sleep disturbance could contribute to cognitive and behavioral impairments, we also tested whether correcting the increased Orx activity is able to restore a normal sleep-wake cycle and alleviate behavioral disturbances. We found that a dual antagonist of Orx 1 and Orx 2 receptors, Suvorexant (Belsomra, MK-4305), which is a new medication for insomnia [38], is able to diminish beta activity intensity, restore the sleep-wake cycle, and alleviate cognitive deficits in R6/1 mice.

\section{Methods}

\section{Animals}

Subjects were male and female F1 mice derived from crossbreeding between male R6/1 transgenic mice [39] (C57BL/6J background, strain number 006471; Jackson Laboratory, Bar Harbor, ME) and female C57BL/6J (Charles Rivers) mice. Tail biopsies were used for PCR genotyping. While the CAG repeat size was not determined for the mice studied in this experiment, the number of repetitions in the breeders was $142.18 \pm 1.195$. At weaning, pups were group-housed with their same-sex littermates and maintained in separate male and female colony rooms under temperature- $\left(22^{\circ} \mathrm{C}\right)$ and humidity-controlled $(50 \%)$ conditions with a $12: 12 \mathrm{~h}$ lightdark cycle (lights on at 7:00 a.m.).

A general outline of protocols was summarized in Table 1 . All experimental procedures were approved by the Institutional Animal Care and Use Committee, Comité d'Ethique pour l'Expérimentation Animale Bordeaux, and were in accordance with the European Communities Council Directive of 24 November 1986 (86/609/EEC).

\section{Drug Administration}

Suvorexant (Belsomra, MK-4305) (Medchem, Princeton, $\mathrm{NL}$ ), a dual antagonist of Orx receptors 1 and 2, was prepared in a solution of 2\% DMSO (Sigma Aldrich, France), 10\% Tween 80 (Sigma Aldrich, France), and water. The same solution, without Suvorexant, was used as the vehicle. The drug was administered orally at a volume of $10 \mathrm{ml} / \mathrm{kg}$. Four doses $(10,30,50,100 \mathrm{mg} / \mathrm{kg}$ ) were tested in experiment 2 (see Table 1) to study the dose effect. The dose of $30 \mathrm{mg} / \mathrm{kg}$ was used in experiments 3 and 4 for sleep recordings and behavioral tests. Suvorexant was injected at 7:00 a.m. in acute experiments (Exp 2 and 3). In the "chronic" treatment, Suvorexant was injected at 7:00 a.m. on 5 consecutive days.

\section{Electrodes and Implantation Surgery}

For EEG recordings, a 4-electrode multisite array that was custom-made in the laboratory was positioned with dental cement on the skull of mice under stereotaxic surgery [10, 14, 40]. For electrodes, silver wires $(130 \mu \mathrm{m}$ diameter) (AM systems Inc, Sequim, WA) whose tips were burned to form spheres were positioned bilaterally above the frontal cortex ( $2 \mathrm{~mm}$ anterior to bregma et and $1 \mathrm{~mm}$ lateral from midline suture) and parietal cortex ( $2 \mathrm{~mm}$ posterior to bregma et and $1 \mathrm{~mm}$ lateral from midline suture). A fifth silver electrode of the same quality was positioned above the cerebellum to serve as both the animal ground and reference. Due to the choice of reference and volume conduction in small mouse brain [41], the beta activity was observed on almost every recorded electrode, in particular from the frontal electrode. Implantation surgery was conducted under $1-1.5 \%$ of isoflurane anesthesia. Mice were also provided with carprofene (Rimadyl, $5 \mathrm{mg} / \mathrm{kg}$ ) for pain relief and inflammation during and after the surgery. Mice were allowed to recover for a week before sleep recordings were performed. 
Table 1 General outline of protocols

\begin{tabular}{|c|c|c|c|c|c|c|c|}
\hline $\begin{array}{l}\text { Exp } \\
\text { no. }\end{array}$ & Study (techniques) & Age & Sex & Genotype & $\begin{array}{l}\text { Experimental } \\
\text { plan }\end{array}$ & Drug dose & Figure \\
\hline Exp 1 & $\begin{array}{l}\text { Orexine activity during light-dark cycle } \\
\text { and disease progression } \\
\text { (immunofluorescence) }\end{array}$ & $2,3,4,5$ months & Male & $\begin{array}{l}\mathrm{WT}(n=50) \\
\quad \mathrm{R} 6 / 1(n=49)\end{array}$ & Independent groups & Not concerned & Figure 1 \\
\hline $\operatorname{Exp} 2$ & $\begin{array}{l}\text { Dose effect of Suvorexant on beta attenuation } \\
\text { (sleep EEG recording) }\end{array}$ & 5 months & Male & $\mathrm{R} 6 / 1(n=9)$ & Repeated measures & $\begin{array}{l}10,30,50 \\
100 \mathrm{mg} / \mathrm{kg}\end{array}$ & Figure 2 \\
\hline Exp 3 & $\begin{array}{l}\text { Effect of Suvorexant on sleep } \\
\text { (sleep EEG recording and behavior) }\end{array}$ & 3 months & Male & $\begin{array}{l}\mathrm{WT}(n=7) \\
\quad \mathrm{R} 6 / 1(n=15)\end{array}$ & Independent groups & $30 \mathrm{mg} / \mathrm{kg}$ & $\begin{array}{l}\text { Figures } 3 \text {, } \\
\quad 4,5\end{array}$ \\
\hline Exp 4 & Effect of Suvorexant on behavior (behavior) & 3 months & $\begin{array}{l}\text { Male } \\
\text { Female }\end{array}$ & $\begin{array}{l}\text { WT }(\mathrm{M} n=5, \\
\text { F } n=5) \\
\text { R6/1 }(\mathrm{M} n=28, \\
\text { F } n=26)\end{array}$ & Independent groups & $30 \mathrm{mg} / \mathrm{kg}$ & $\begin{array}{l}\text { Figures } 6 \text {, } \\
\quad 7\end{array}$ \\
\hline
\end{tabular}

\section{Electrophysiological Recordings During Sleep and Data Analysis}

Mice were handled and habituated to the recording chamber and room for $48 \mathrm{~h}$ prior to each EEG recording unless otherwise mentioned. Recordings were performed for either 3-4 h during the light period for the dose effect experiment (Exp 2) or $24 \mathrm{~h}$ starting immediately after injections for sleep behavioral (Exp 3) studies.

Recordings were performed using five copies of our custom-made EEG recording system integrating "OpenBCI" data acquisition card (https://openbci.com). This new system consists of a low-cost and compact acquisition card initially designed for EEG recordings in humans. The card is set up for both signal amplification and analog-digital conversion with $2-\mathrm{kHz}$ sampling rate, and storage on a mounted micro-SD memory card. This small and lightweight card to which mice were connected using flexible and lightweight cables $(50-\mu \mathrm{m}$ copper wires inserted into a silicon tube) was suspended above the animals using a custom-made rotative commutator. The rotative commutator securing free movement of the mouse by preventing the cable twisting was a magnetic trust bearing fixed onto a metallic frame placed above the board. The very low torque of the magnetic bearing enabled following passively the board suspended under it via a stainless needle and a thread, which by itself, passively follows the rotations of the tethered mouse moving below the board. The entire system is constructed in the laboratory.

Recorded EEGs were visualized for inspection using Sonic Visualiser (www.sonicvisualiser.org) to manually delimit the timing of various vigilance states by taking into account EEG spectra as previously described $[10,14]$. A naive experimenter performed this operation. The specific segments of EEG attributed to different brain states were then processed for averaged power spectra using the Matlab platform with laboratory-built programs. Fast Fourier transforms were calculated on 4-s Gaussian windows with $50 \%$ overlap. Because the intensity of the spectra might differ between probes and animals, intensities at different frequencies were normalized by the sum of the overall spectra before being averaged by genotype, treatment, etc., for data presented in Figs. 2 and 3.

To provide a fine and objective quantification of beta power changes under Suvorexant against vehicle treatment within each animal, we fitted a Gaussian peak on a decaying exponential background model to our raw spectral power distribution for each vigilance state and treatment. The resulting fitted curve corresponded with the sum of the exponential decay and Gaussian distribution. The amplitude and surface of the Gaussian distribution appearing in the ranges of $20-40 \mathrm{~Hz}$ allowed the estimation of peak and area of beta power, respectively. The differences of these values (amplitude and area) between the two treatments (vehicle and Suvorexant) were analyzed statistically using one-sample $t$ test with hypothesized mean $=0$ (i.e., no difference between vehicle and Suvorexant treatments).

\section{Behavioral Assessment: Body Weight, Y-Maze, Rotarod, Nest Construction}

At 3-3.5 months of age, mice were placed in standard individual holding cages $30 \mathrm{~min}$ before the first test and were left undisturbed in the experimental room to allow them to acclimatize to the new environment. Mice were first tested for spatial recognition memory in the Y-maze followed by motor coordination and motor learning task in the rotarod test (three trials). After this last test, mice returned to the colony room overnight for the nest building test in their individual cages.

\section{Body Weight}

Mice were weighed on the day of injections for acute pharmacological treatment experiment. In the 5-day treatment experiment paradigm, they also were weighed again on the 5th day, just before the last treatment. 


\section{Y-Maze}

Spatial recognition memory was tested in a Y-maze constructed in gray Plexiglas. The maze was placed on a table $80 \mathrm{~cm}$ high and located in a room containing extramaze visual cues. The three arms $(42 \times 8 \times 15 \mathrm{~cm})$ of the Ymaze were similar in appearance and dimension, and spaced at $120^{\circ}$ from each other. The mouse's locomotion was tracked and analyzed via a camera placed above the maze using Ethovision 9 (Noldus Technology, Wageningen, The Netherlands). Mice were arbitrarily assigned two arms (start and familiar arms) to which they were exposed during the first phase of the test (sample phase). The remaining third arm, blocked by a transparent door placed at the entrance, constituted the novel arm for the second phase. Allocation of arms (start, familiar, and novel) was counterbalanced within each experimental group. During the sample phase, mice were placed at the end of the designated start arm and allowed to explore freely both the start and the other unblocked (familiar) arm for $5 \mathrm{~min}$. Mice were then removed from the maze and returned to the waiting cage for $10 \mathrm{~min}$ of retention interval, before the test phase. During the test phase, the door blocking the "novel arm" was removed. Mice were placed at the end of the same start arm and allowed to explore the entire maze for $2 \mathrm{~min}$. The timing of the 2-min test phase period was initiated only once the mouse had left the start arm. In the interval between the sample and the test phases, the apparatus was cleaned with alcohol and water to remove odor residue. Time spent in each arm of the maze was analyzed and performance on the test phase was evaluated by the time spent in the novel arm in comparison with the time spent in the familiar arm. A preference index was calculated as the time spent in the novel arm divided by the time spent in the 3 arms $\times 100$.

\section{Rotarod Test}

Motor coordination and balance of mice were assessed in the standard accelerating rotarod apparatus (Imetronic, Pessac, France), with the rotating speed increasing from 3 to 18 rotations per minute every $30 \mathrm{~s}$ during the 3-min trial. Mice were given 3 testing trials with a 10 -min interval. The latency to fall from the beam, with a maximum of $3 \mathrm{~min}$, was recorded.

\section{Nest building test}

Mice in their individual cages were then provided with a Nestlet (Bellmore, USA) overnight. The nest quality was assessed the next morning (8:00-9:00 a.m.) on a rating scale of 1-5 (1, cotton not touched; 5 , perfect and tridimensional nest) according to the standard scoring technique [42].

\section{Preparation of Brains}

Male R6/1 and WT littermate mouse brains were collected at 2, 3, 4, and 5 months of age. Half of each genotype and age group were sacrificed at 5:30-7:00 a.m. (active period) and the other half were sacrificed at 5:30-7:00 p.m. (inactive period). Mice were deeply anesthetized with an overdose of sodium pentobarbital $(100 \mathrm{mg} / \mathrm{kg}$, i.p.) and transcardially perfused with $4 \%$ paraformaldehyde solution. Brains were stored overnight in the same fixative solution and then transferred to $30 \%$ sucrose solution for an additional $72 \mathrm{~h}$ before being cut into $50-\mu \mathrm{m}$-thick coronal sections on a freezing microtome (Leica, Mannheim, Germany). Sections were kept at $-20{ }^{\circ} \mathrm{C}$ in a cryoprotectant solution (30\% glycerol $(v / v)$ and $30 \%$ ethylene glycol $(v / v)$ in $0.1 \mathrm{M}$ phosphate buffer ( $\mathrm{PB}, \mathrm{pH}$ 7.4)) until being processed for immunofluorescence.

\section{Immunofluorescence: c-Fos, Orx, and MCH in Neurons in the Lateral Hypothalamus}

One of every six sections containing the lateral hypothalamus was stained for multiple immunofluorescence labeling to identify orexin- or MCH-expressing neurons and to detect c-Fos expression in these neurons. Free-floating coronal sections were first incubated for $1 \mathrm{~h}$ in blocking PBS solution containing 4\% donkey serum and $0.3 \%$ Triton X-100 (Sigma, St. Quentin Fallavier, France). They were then incubated for $72 \mathrm{~h}$ at $4{ }^{\circ} \mathrm{C}$ with primary antibodies diluted with the blocking solution. Antibodies were raised in rabbit against $\mathrm{MCH}$, generously provided by Dr. P.Y. Risold, (Université de Franche-Comté, Besançon, France, 1:1500), in goat against Orx (Santa-Cruz Biotechnology, sc$8070,1: 500$ ), and in guinea pig against c-Fos (Synaptic System, 226004, 1:1000). The sections were then rinsed in PBS and incubated for $1 \mathrm{~h}$ in blocking solution containing secondary antibodies as follows: Alexa Fluor-488 donkey anti-goat, Alexa Fluor-568 donkey anti-rabbit, and Alexa Fluor-647 donkey anti-guinea pig (1:500; Jackson Immunoresearch). The sections were washed, mounted on glass slides, and cover slipped. Observation and acquisition were performed with a Leica DM6000B microscope (Leica Mannheim, Germany) equipped with a Qimaging RETIGA.

Mosaic image stacks of the whole lateral hypothalamus structure on right and left hemispheres were acquired using micromanager to control the acquisition process with an oil immersion $\times 20$ lens. For each lateral hypothalamus, a stack of 5 images with a step of $2 \mu \mathrm{m}$ were acquired, and the number of Orx neurons and $\mathrm{MCH}$ neurons was counted using the ImageJ software. Moreover, the number of colocalized Orx neurons with c-Fos and colocalized MCH neurons with cFos was counted, enabling the percentage of active (i.e., cFos expressing) Orx and MCH neurons, respectively. All analyses were performed by an experimenter blind to the genotype and age. 


\section{Data Analyses and Statistics}

If raw electrophysiological, behavioral, and immunocytochemical data or their transformed data (log, square root) were normally distributed and had homogenous variance, data were analyzed using parametric $t$ test or ANOVA tests. Otherwise, non-parametric Mann-Whitney or Kruskal-Wallis tests were performed. Discriminant analysis was performed to verify if the linear combination of different measures/variables was sufficient to assign a mouse into treatment versus vehicleinjected group. Analyses were performed using Statistica, GraphPad Prism, and Statview softwares. Data were presented either mean \pm SEM (for parametric tests) or median \pm minmax range (for non-parametric tests).

\section{Results}

\section{Experiment 1: Orexinergic Activity During Circadian Cycle and Aging in R6/1 Mice}

Orx and $\mathrm{MCH}$ neurons play reciprocal roles across the sleepwake cycle [43]. To characterize Orx and $\mathrm{MCH}$ activity during the circadian cycle across age and development of the disease, male R6/1 and WT littermate mouse brains were collected at 2, 3, 4, and 5 months of age. Half of each genotype and age group were sacrificed between 5:30 and 7:00 a.m. (active period) and the other half were sacrificed between 5:30 and 7:00 p.m. (inactive period). Triple immunofluorescence was conducted using antibodies against c-Fos, Orx, and $\mathrm{MCH}$. The percentage of neurons expressing c-Fos was calculated against counted Orx- or MCH-expressing neurons as an index of their activity. Orexin- and $\mathrm{MCH}$-expressing neurons are known to form distinct populations in the lateral hypothalamus $[44,45]$. As previously reported in rats [46], a negligible proportion of $\mathrm{MCH}$ neurons were found to be cFos+ in both WT and R6/1 mice across ages, which prevented further analysis to be performed.

Three-factor ANOVA involving genotype, age, and lightdark period factors indicated that Orx activity was generally higher in the active (dark phase for rodents) than in the inactive period (light phase) $(F(1,83)=47.64, p<.0001$, Fig. 1). However, no main age- $(F(3,83)=2.341, p=.079)$ or genotype-related effects $(F(1,83)<1$, n.s. $)$ were observed. On the other hand, the same analysis revealed that the lightdark period-associated activity did depend on genotype (period $\times$ genotype interaction, $F(1,83)=21.278, p<.0001)$, age (period $\times$ age interaction, $F(3,83)=11.759, p<.0001)$, and both age and genotype (period $\times$ genotype $\times$ age interaction $F(3,83)=3.504, p=.0189)$. Further post hoc pairwise comparisons revealed, as expected, higher levels of Orx activity in the active period, compared to the inactive one, at all ages studied in WT mice (all $p<.05$ ). However, in R6/1 mice, this difference was observed only at 2 months $(p<.0001)$, and was not anymore present at 3 and 4 months ( $p>.05$ for both ages). Interestingly, at 5 months of age, Orx activity in the active period was significantly lower than in the inactive period $(p=.016)$, a pattern that was opposite to that expressed by age-matched WT or their younger counterparts (2 months).

\section{Experiment 2: Antagonizing Aberrant Orx Activity Reduces Beta Activity in Symptomatic R6/1 Mice}

The increased Orx activity observed during the inactive period of R6/1 mice, which became accentuated at 5 months, led us to suspect its association with the beta activity that was also specifically intense during the inactive sleep periods. We therefore asked whether inhibiting the aberrant Orx activity in the inactive period by the administration of a selective dual antagonist for both Orx 1 and Orx 2 receptors, Suvorexant, could modify beta activity occurrence and power. Fivemonth-old R6/1 mice received one oral administration of Suvorexant at $30 \mathrm{mg} / \mathrm{kg}$ before being subjected to EEG recordings for 3-4 h. The dose of $30 \mathrm{mg} / \mathrm{kg}$ was determined in a preliminary study of dose-dependent effects (data not shown).

The EEG recordings in these experiments revealed that the beta activity power in R6/1 mice during REM sleep was considerably attenuated by Suvorexant, while the power and frequency of theta oscillations also expressed in this state in both genotypes remained unchanged (Fig. 2 a). We then quantified peak amplitude and surface of beta frequencies by fitting a Gaussian peak on a decaying exponential background model to our raw spectral power distribution for each animal, vigilance state, and treatment. Data expressed as differences between vehicle and Suvorexant treatments within animals revealed that Suvorexant attenuated $71.71 \pm 7.79 \%$ in amplitude, and 71.71 $\pm 8.42 \%$ in surface area of beta activity during REM sleep. These values were significantly different from 0 , i.e., the absence of attenuation (one-sample $t$ test, $t(6)=9.208, p<.0001$ for amplitude, $t(6)=8.517, p=.0001$, for surface). Although the beta activity remained less intense during SWS sleep, a similar beta attenuation $(78.14 \pm 8.31 \%$ in amplitude and $75.73 \pm 9.63 \%$ in surface) was also found during this period (Fig. 2 b, $t(6)=9.399, p<.0001$, for amplitude $t(6)=7.834$, $p=.0002$ for surface). Because animals did not reliably exhibit beta activity in physiological condition, the same analysis was not performed for wake spectra.

\section{Experiment 3: Orx Antagonism Attenuates Beta Activity and Improves Sleep in Early Symptomatic R6/1 Mice}

We then examined if Suvorexant might also be effective in attenuating the beta and restore the disturbed sleep-wake cycle power in younger, early symptomatic (3-3.5-month-old) R6/1 mice. Therefore, we performed EEG recordings for $24 \mathrm{~h}$ 
Fig. 1 Orexin neuron activity during active (dark) and inactive (light) behavioral periods in R6/1 and WT littermates from 2 to

5 months of age. a Representative coronal section of the lateral hypothalamus from a 2-monthold WT mouse sacrificed during its active period and labeled for $\mathrm{MCH}$ (gray), orexin (green), and c-Fos (red). The image shows large groups of Orx-positive neurons (white arrow) surrounded by $\mathrm{MCH}$-positive cells (white arrowheads). While c-Fos was detectable in a number of Orxcontaining neurons (empty arrowheads), only a negligible proportion $(<1 \%)$ of $\mathrm{MCH}$ neurons were c-Fos+. White frame detailed in right insets displays MCH-, orexin-, c-Fosand merge-labeling. V3, third ventricle. Scale bar, $100 \mu \mathrm{m}$. b Data $($ mean $\pm \mathrm{SEM})$ were expressed as mean percentage of c-Fos immunoreactive neurons among Orx immunoreactive cells during the active (gray bars) or inactive (white bars) periods. ${ }^{*} p<.05, * * * p<.001$, $* * * * p<.0001$ by unpaired $t$ test
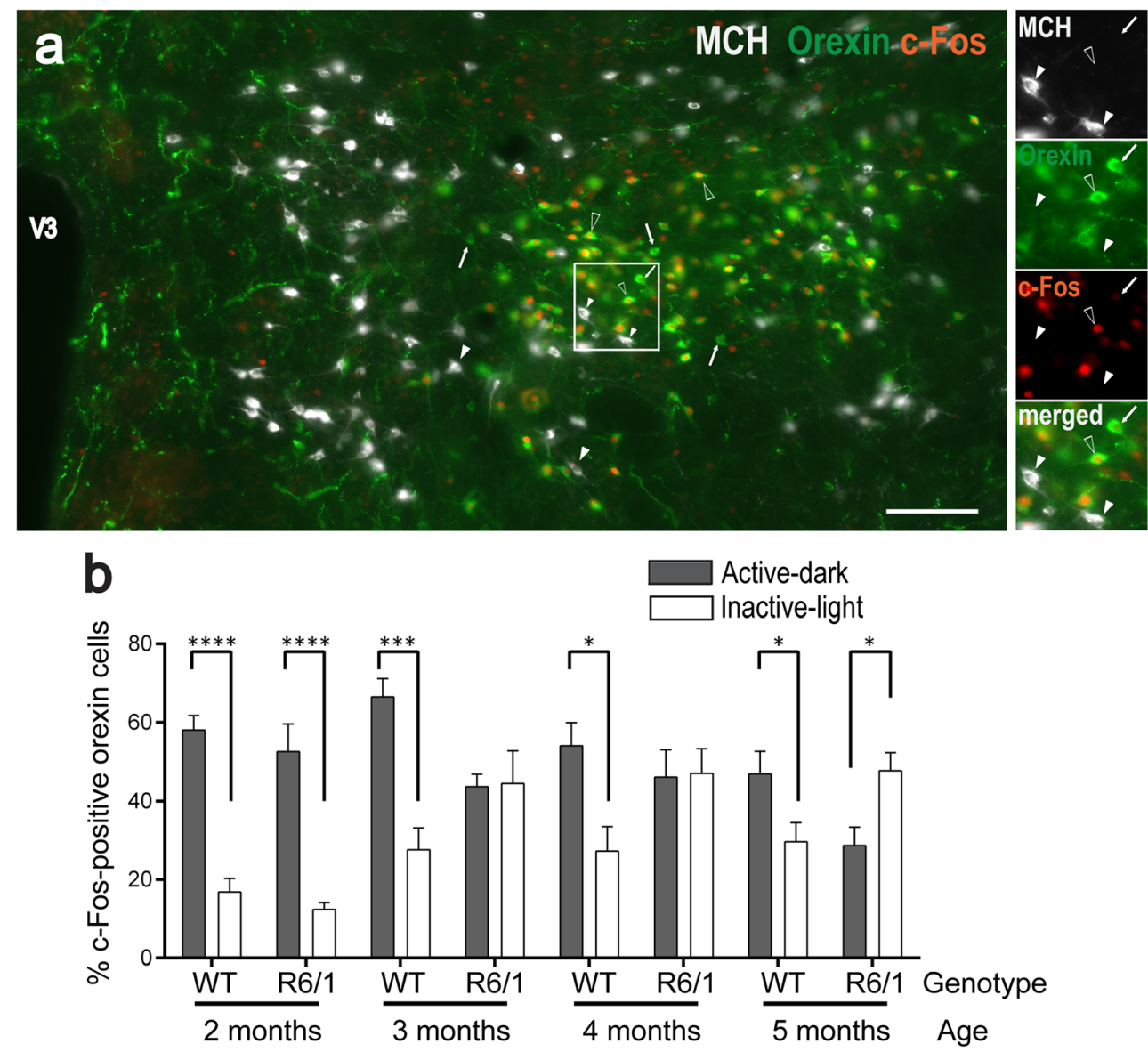

following a single injection of Suvorexant in the morning (7:00 a.m., at the beginning of the inactive period) in both R6/1 and WT littermate mice.

Firstly, spectral analyses of REM sleep EEG showed that the intensity of the beta activity, which is present at these ages, was reduced following the administration of Suvorexant in R6/1 mice (Fig. 3 a). The beta attenuation reached $68.75 \pm 8.39 \%$ in amplitude and $65.00 \pm 10.01 \%$ in surface during REM sleep. These attenuations were statistically significant (by one-sample

Fig. 2 Effects of Suvorexant on beta activity $(20-35 \mathrm{~Hz})$ in 5 month-old R6/1 mice. Plots of average power spectra showing that a single oral administration of Suvorexant $(30 \mathrm{mg} / \mathrm{kg}$ ) decreased the power of the beta activity during REM sleep (a) and SWS (b) but not wakefulness (c). Theta (6-10 Hz) power strongly expressed during REM sleep was not modified (a). Data are presented as mean \pm SEM

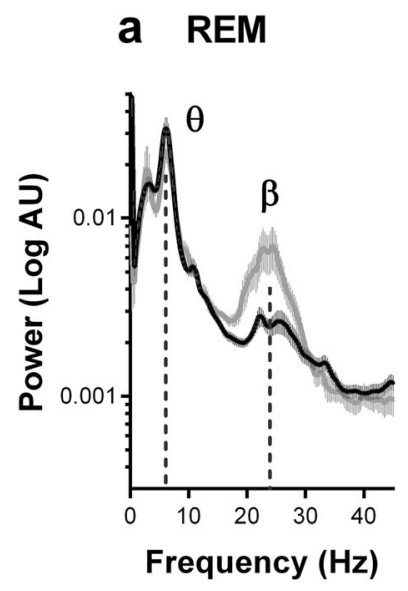

$t$ test, $t(6)=8.195, p<.0001$, and $t(7)=6.492, p=.0003$ for amplitude and surface, respectively). A similar beta attenuation was also observed during the SWS $(75.25 \pm 9.96, t(7)=7.55$ $p=.0001$ in amplitude, $75.78 \pm 10.25, t(7)=7.357, p=.0002$ in surface, Fig. 3 b). Once again, data for wake spectra were anymore analyzed, as a substantial proportion of animals did not display quantifiable beta activity.

Secondly, as could be seen in representative hypnograms (Fig. 4), sleep behavioral analysis revealed that blocking the

\section{b sws}

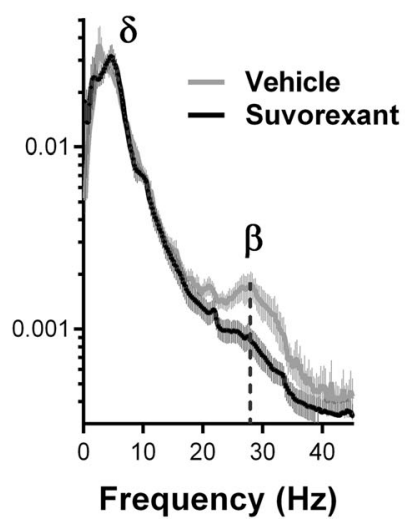

C Wake

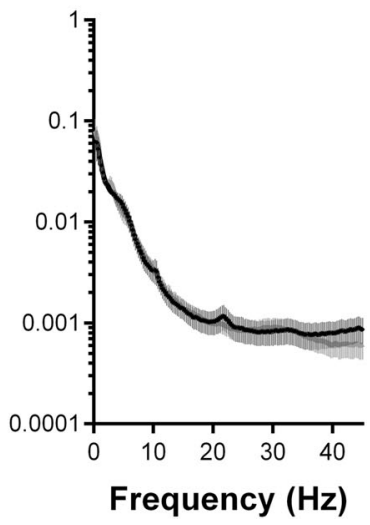


Fig. 3 Spectral changes of frontal EEG following Suvorexant treatment in 3.5-month-old R6/1 mice during REM sleep (a) and SWS (b). Beta activity power was significantly decreased. Data are presented as mean \pm SEM a R6/1 REM

b R6/1 SWS

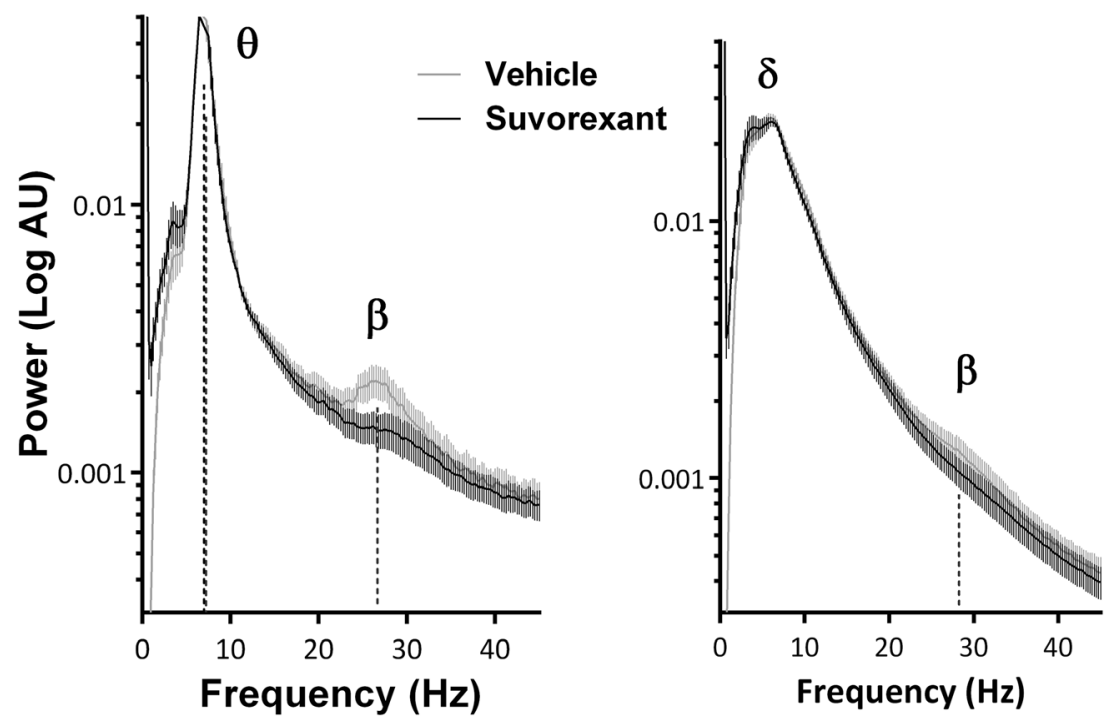

Orx activity with Suvorexant during the inactive period significantly reduced SWS and REM sleep-onset latencies in R6/ 1 mice (Fig. 5 a, b) ( $p=.0055, p=.0078$ for SWS and REM sleep, respectively by Mann-Whitney) compared to vehicletreated counterparts. This decrease was not significant in WT mice $(p=.157)$ (Fig. 5 a, b). In addition, whereas vehicletreated WT mice, as expected, spent more time awake during the active dark period than in the inactive light period $(p<.05)$, their $\mathrm{R} 6 / 1$ counterparts remained awake to a similar extent during both periods (Fig. $5 \mathrm{c}, p>.05$ ). While this pattern of activity, in general, was not modified by Suvorexant treatment in the transgenic (and WT) mice, their percentage of time spent in REM sleep in the inactive period was significantly increased $(t(13)=-2.196, p=.047$,$) , and percentage in$ SWS had tendency to increase $(p=.07)$, and percentage in wake had tendency to decrease ( $p=.07)$.

Thirdly, blocking Orx receptor activity significantly increased the number of waking, SWS, and REM sleep episodes in R6/1 mice during the inactive period ( $p<.05$ for all, by $t$ test) (Fig. $5 \mathrm{~d}$ ) and the number of waking episodes in WT counterparts $(p<.05)$. The increased number of wake episodes in both genotypes, however, did not lead to increased wake durations, suggesting fragmented sleep-wake activity under Suvorexant treatment. This tendency was more pronounced in WT mice as the augmented numbers of SWS and REM sleep did not modify percentage of durations (Fig. 5 c, d).

\section{Experiment 4: Orx Antagonism Alleviates Behavioral Impairments in a Subpopulation of R6/1 Mice}

In R6/1 mice, significant behavioral impairments emerge at young ages (3-3.5 months) $[10,47,48]$. Since an acute injection of Suvorexant ameliorates sleep and attenuates beta activity in R6/1 mice, we then tested whether these neural activity and sleep changes may affect behavioral performance in R6/1 mice. Male R6/1 mice were acutely injected with either
Fig. 4 Representative hypnograms of 3.5-month-old male WT and R6/1 mice. Hypnogram of vehicle-treated WT (a), Suvorexant-treated WT (b), vehicle-treated R6/1 (c), and Suvorexant-treated R6/1 (d) mouse. Note that Suvorexanttreated mice of both genotypes (b, d) fell asleep immediately after Suvorexant injection a

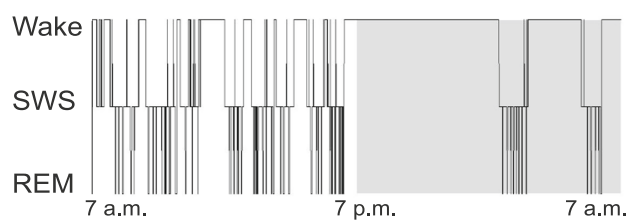

C

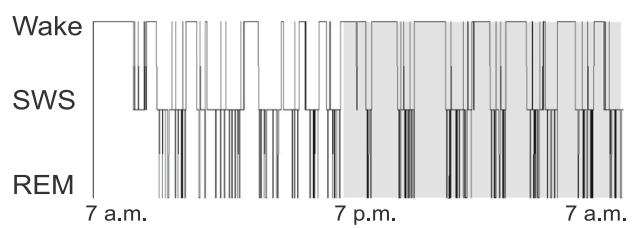

b

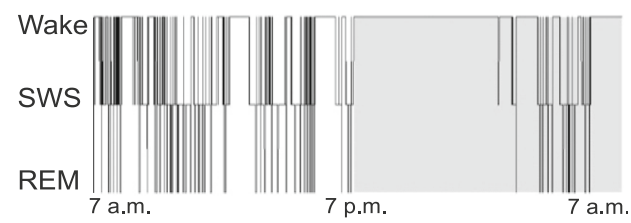

d

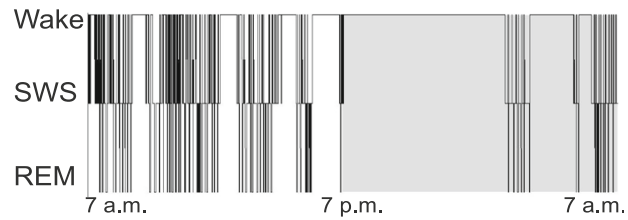


Fig. 5 Effects of Suvorexant on sleep in 3.5-month-old male R6/1 and WT littermates. Sleep-onset latencies to first SWS (a) and REM sleep (b) episodes following Suvorexant treatment. Percentage of durations (c) and number of episodes (d) of sleep and waking during inactive light and active dark periods following treatment. Data are presented as median \pm min-max range in $\mathbf{a}$ and $\mathbf{b}$ and mean \pm SEM in $\mathbf{c}$ and $\mathbf{d}$. $* p<.05, * * p<.01, \# p=.07$

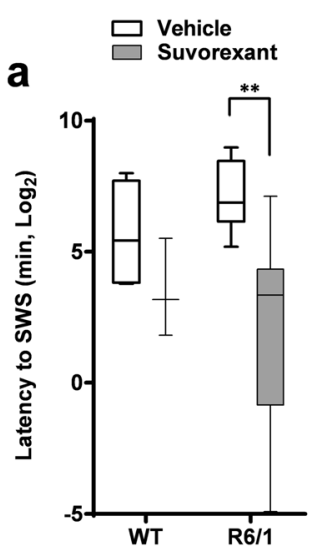

C

b

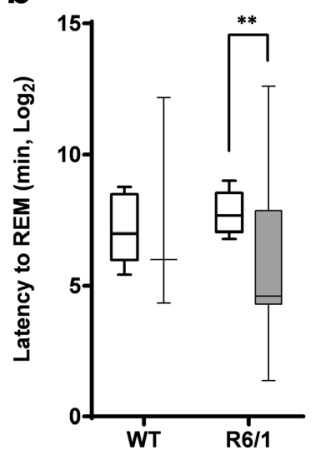

d
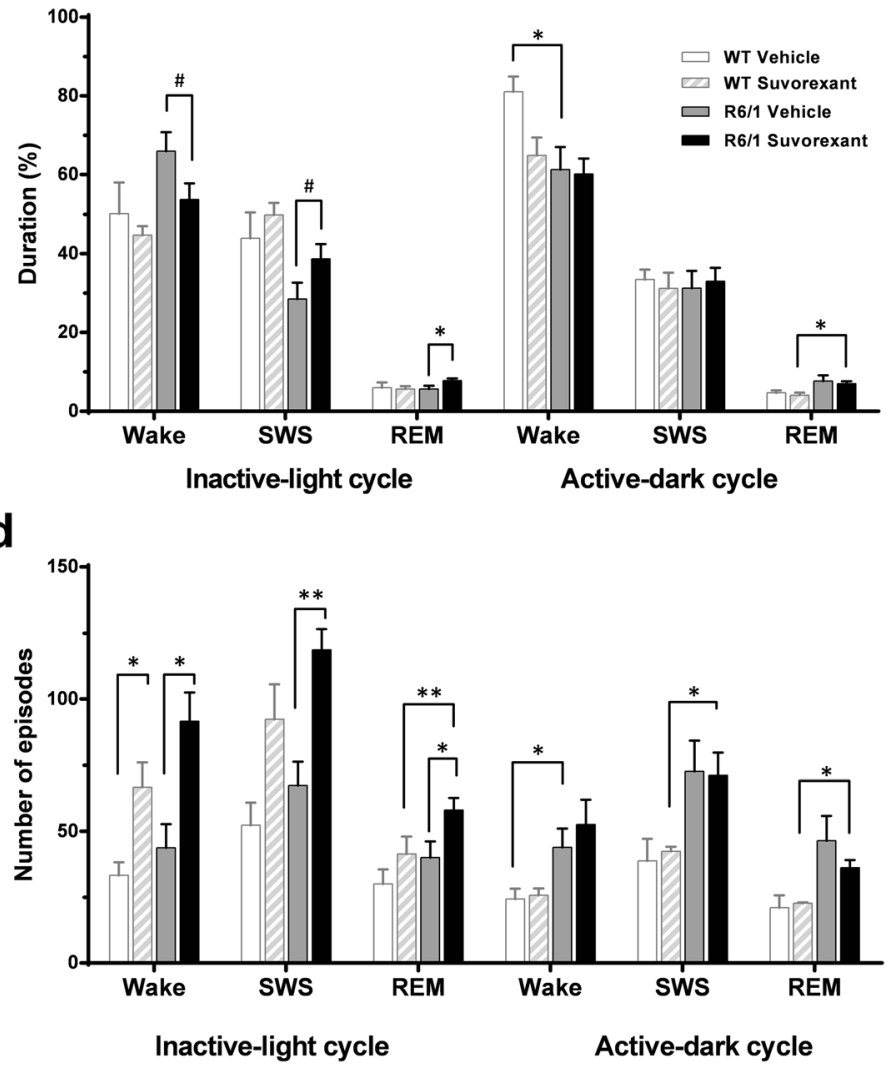

Suvorexant or vehicle at 7:00 a.m. and allowed to sleep. Behavioral testing was then conducted $12 \mathrm{~h}$ post-injection at 7:00 p.m., the beginning of the following active period. Mice were tested for spatial recognition memory in a Y-maze, motor coordination in a rotarod, and nest building capability. Because our results showed that there was no improvement in the performances of these three behavioral tests (data not shown), we concluded that a single injection of Suvorexant was unable to alleviate behavioral deficits in 3-month-old male R6/1 mice.

We then tested whether Suvorexant injections over a prolonged period of 5 days might be effective for improving behavioral performances. R6/1 female mice were also included in addition to R6/1 male mice in this study because of the importance of gender differences reported in $\operatorname{HD}[15,49,50]$. Animals were administered drug for 5 consecutive days at 7:00 a.m. daily, and tested in the above behavioral tasks on the 5 th day, $12 \mathrm{~h}$ after the last injection (7:00 p.m.). The results indicated that both male and female Suvorexant-treated R6/1 mice had gained weight by the end of the 5-day treatment compared to vehicle-treated counterparts (treatment effects, $F(1,51)=7.167, p=.01)$ (Fig. 6 a), (gender $\times$ treatment interaction, $F(1,51)<1$, n.s.). Such weight gain effects were not found in WT mice $(F(1,12)<1$, n.s., data not shown). Moreover, female R6/1 mice, irrespective of treatment, gained more weight than male mice $(F(1,51)=12.196, p=.001)$ (Fig. 6 a). However, no beneficial effects of Suvorexant were found for motor coordination scores averaged from 3 trials (effect of treatment $U=318, U^{\prime}=410$, n.s.) (Fig. 6 b), although female R6/1 mice generally displayed higher scores than male counterparts (effect of sex $U=169, U^{\prime}=559$, $p=.007$ ) (Fig. $6 \mathrm{~b}$ ). No such gender difference was observed in WT mice ( $p>.05$, data not shown).

Furthermore, no significant differences in the quality of nest construction were found between vehicle- and Suvorexant-treated R6/1 mice, irrespective of gender ( $U=$ $266.5, U^{\prime}=381.5$, n.s.), although female R6/1 mice, irrespective of treatment, constructed higher quality nests than males ( $U=193.5, U^{\prime}=456.5, p=.013$ ) (Fig. $6 \mathrm{c}$ ), while being impaired as compared to WT females $(p<.05)$. Finally, Suvorexant-treated R6/1 mice, as compared to vehicletreated counterparts, displayed overall higher spatial recognition memory (main effect of treatment, $F(1,47)=4.651$, $p=.036$ ), although importantly, this was mainly due to the superior performances of female R6/1 mice (treated with Suvorexant) $(p<.05)$, rather than $\mathrm{R} 6 / 1$ males in which such improvement was not found $(p>.5)$ (gender $\times$ treatment interaction $F(1,47)=9.494, p=.0034)$ (Fig. $6 \mathrm{~d}$ ).

Discriminant analysis assessed whether the linear combination of the previous behavioral scores (summarized in Fig. 6) is 
a
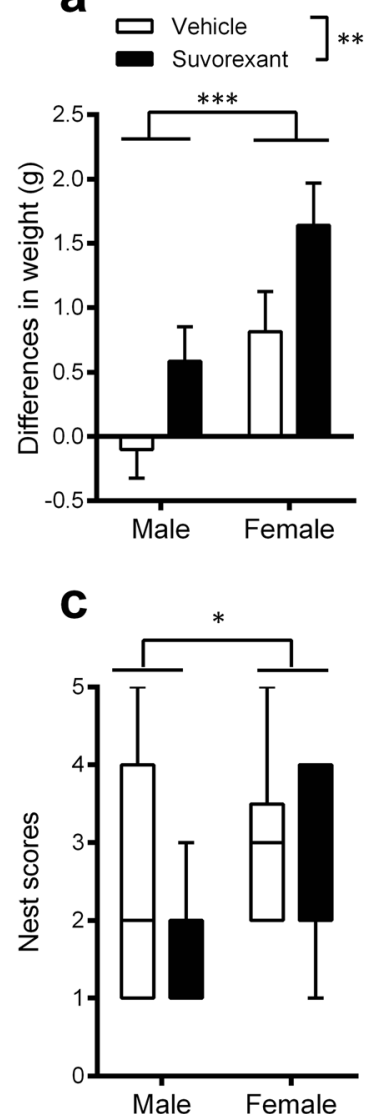

b

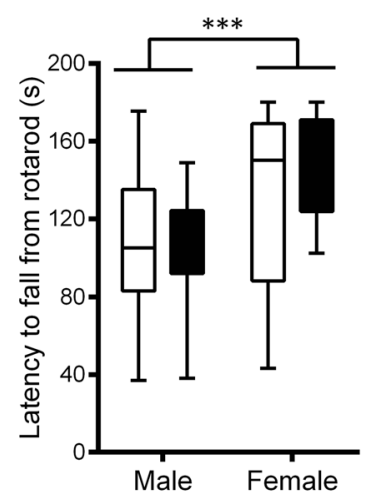

d

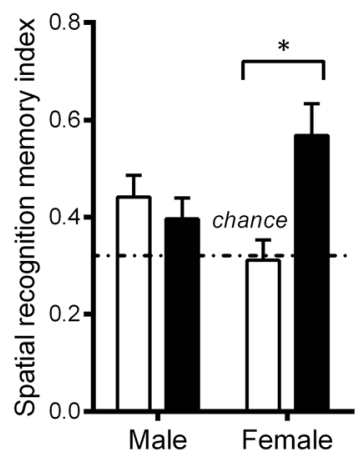

Fig. 6 Effects of a 5-day administration of Suvorexant on behavioral performances in 3-month-old R6/1 mice of both genders. Body weight (a), motor coordination in a rotarod (b), nest construction (c), and spatial recognition memory in a Y-maze (d) in male and female R6/1 mice. Data are presented as mean \pm SEM in $\mathbf{a}$ and $\mathbf{d}$, median \pm min-max range in $\mathbf{b}$ and c. $* p<.05, * * p<.01, * * * p<.001$

effective in predicting whether an animal belongs to the Suvorexant or vehicle R6/1 group for each gender. This analysis segregated individual R6/1 females into Suvorexant- versus vehicle-treated group with a success rate of $76.9 \%$ (Lambda Wilks $.53473, F(7,18)=2.2374, p=.079)$. Therefore, the discriminant analysis assigned 9 out of $13(69.2 \%)$ vehicle-treated R6/1 mice by classifying them to the vehicle group and assigned 11 out of 13 (81.8\%) Suvorexant-treated mice classified in the Suvorexant-treated group successfully (Fig. 7 b). Only four vehicle-treated and two Suvorexant-treated mice were classified into the wrong groups. Suvorexant-treated female mice, therefore, showed a small Mahalanobis distance to the centroid of the Suvorexant-treated group and a large distance to the centroid of the vehicle-treated group, and vice versa for the vehicle-treated female mice. In contrast to female mice, the discriminant analysis assigned only $64.3 \%$ of all males to the correct groups (Lambda Wilks $.82474, F(7,20)=0.60717, p<.7433$, Fig. 7 a), thus preventing a significant discrimination between the experimental groups to be made. This finding therefore further supports the

conclusion that a 5-day administration of Suvorexant induced an alleviation of behavioral deficits in female R6/1 mice.

\section{Discussion}

In this study, we have investigated whether R6/1 mice also display Orx activity alteration with respect to activityinactivity cycle, and if so, the modified Orx activity could be linked to abnormalities of HD-associated neural synchrony, sleep architecture, and cognitive and motor performances. Our data demonstrate that the Orx system in R6/1 mice begins to display an altered activity pattern, revealed by c-Fos imaging technique, at 3 months, the age at which behavioral disturbances appear in this mouse model [10, 47, 48]. Specifically, our data show that, at this age, Orx activity is characterized by an abnormal increase in activity during the animal's inactive period, leading to an indistinguishable inactivity-activity cycle. At 5 months, Orx activity further deregulates as it reverses completely compared to that of the regular inactivity-activity cycle displayed by WT mice. These results confirm those previously observed in R6/2 mice [37], and further document the progressive deregulation of Orx activity pattern across aging in R6/1 mice.

Antagonizing increased activity of the orexinergic system during the transgenic animal's inactive period, by administering Suvorexant, a dual antagonist of Orx 1 and Orx 2 receptors, produced beneficial consequences at several levels. First, pharmacological inhibition of the Orx activity significantly attenuated the HD associated beta synchrony in R6/1 mice. Second, the Orx antagonism during the inactive light period alleviated the sleep-wake abnormalities, with R6/1 mice exhibiting (1) decreased sleep latencies, (2) increased amount of sleep and reduced waking, (3) and also a fragmentation of sleep, and this additionally in WT mice. These transient modifications during the inactive period therefore tended toward restoring the normal sleep-wake pattern. Third, Orx inhibition during 5 consecutive days attenuated weight loss in both male and female R6/1 mice, with R6/1 females additionally displaying alleviated cognitive deficits.

The dysregulated activity pattern of Orx neurons at 3 months was found to coincide with the appearance of the beta activity and sleep disturbances in R6/1 mice. The administration of the Orx receptors antagonist during the inactive behavioral period of R6/1 mice was associated with the attenuation of the power of the beta activity and the restoration of the sleep-wake cycle to some extent. Significantly, other studies have reported that a single administration of hypnotic drugs acting on the GABAergic or noradrenergic systems, or anti-depressant substances, also decreases the amplitude of the beta/gamma activity in R6/2 mice [51, 52], while such data are not available in the R6/ 1 model. However, hypnotic drugs also affect other physiological brain waves that are not associated with HD pathology, such as 
Fig. 7 Discriminant analysis in male (a vehicle-, Suvorexanttreated) and female (b vehicle-, Suvorexant-treated) R6/1 mice at 3 months of age. Each dot represents a R6/1 mouse distributed in Mahalanobis distance from the centroid of the vehicle and Suvorexant groups a Male

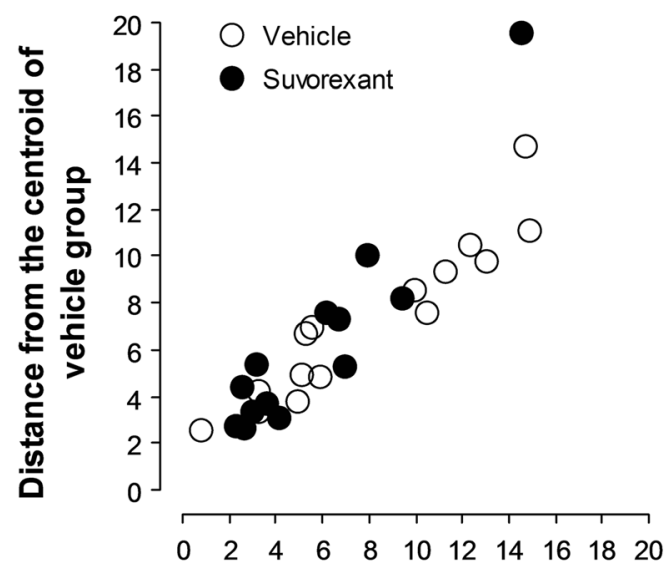

Distance from the centroid of suvorexant group b Female

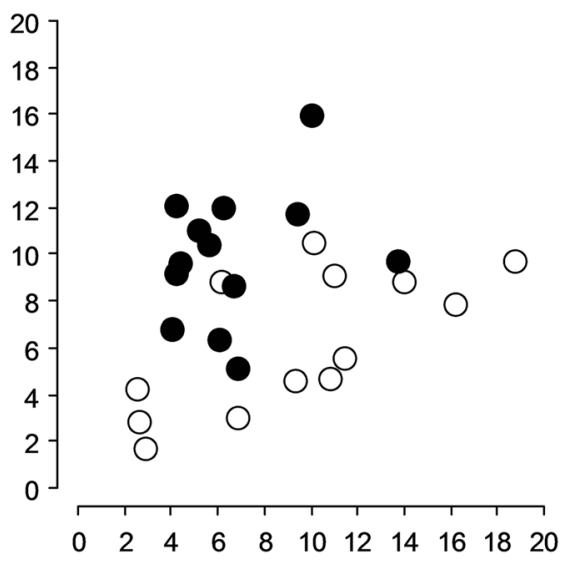

Distance from the centroid of suvorexant group theta activity expressed typically during REM sleep by increasing theta power and decreasing its frequency both in rodents [53] and humans [54]. Our work further shows that antagonizing Orx 1 and Orx 2 receptors affects only the pathological beta activity by decreasing its power without changing other brain waves. These results support the importance of the Orx system in normal sleep-wake cycle and its association with the altered EEG of HD mice. However, further studies are required to assess the exact role of the Orx system in HD pathogenesis and whether the beneficial effect of Suvorexant is due to a direct mechanism or action.

In fact, it is unclear whether the Orx system malfunction in our R6/1 mice is due to afferent or efferent pathway abnormalities. Previous studies have reported that at least some of the afferent pathways to the lateral hypothalamus area seem to function normally in the R6/2 and Q175 models [13, 55]. For example, although circadian clock genes are dysregulated in R6/2 mice, the intrinsic membrane properties of suprachiasmatic neurons, which integrate these altered genes and project to Orx neurons, remain functional in vitro, i.e., outside the pathological circuit [55]. However, a recent study has reported early functional alterations of suprachiasmatic neuron in BACHD and Q175 mice [56]. One work has also suggested that downstream nuclei in the brainstem or the hypothalamus to which Orx neurons project are intact in HD mouse models [17], but once again, no data are available for the R6/1 model. Therefore, it remains possible that functional impairments to Orx receptors expressed in cortical and other brainstem regions may be at the origin of the abnormality observed here. Further studies are required to locate the source of beta activity in the HD mouse brain and to determine whether the Orx activity alterations may play a key role in the generation of this HD-specific network activity.

The behavioral effects observed in our HD mice could result from an Orx inhibition of sleep-mediated mechanisms, or an impact on other physiological processes also regulated by the Orx system, such as stress, reward, appetite, and glucose metabolism $[57,58]$. Indeed the latter possibility was supported by the finding that five consecutive daily injections significantly increased body weight in our R6/1 mice. In this context, it is known that Orx is also involved in the control of feeding behavior, metabolism, and overall energy balance. Orx signaling promotes food-seeking behavior in response to short-term fasting in order to increase food intake and thus maintain body weight. However, the Orx control of body weight is more complex, since it also supports energy expenditure to protect against obesity. It has been reported that transgenic mice in which Orx neurons are ablated show feeding abnormalities and a dysregulation of energy homeostasis that lead to obesity, despite a reduction in food intake [59]. A more recent study found that female Orx KO mice display a significant increase in body weight, whereas an equivalent weight variation is not observed in males [60]. In our study, the administration of an Orx receptor antagonist to R6/1 mice led to a body weight increase both in males and females, although the latter group gained more weight, further indicating the existence of a gender difference in the Orx control of metabolism. Also supporting this idea has been the finding of differing Orx 1 and 2 receptor levels in male rats compared to females, consistent with a gender difference in actual Orx neurotransmission [61]. Furthermore, female, but not male, rats display an increased activation of Orx neurons and an associated increase in feeding during rebound from $48 \mathrm{~h}$ fasting [62]. Moreover, a positive correlation between body weight, fat gain, and increased activation of Orx neurons in female, but not male mice, has been reported [63]. However, in the present study, we did not detect any gender differences in body weight for WT mice, suggesting that additional studies will be required to understand Orx's complex regulation of energy metabolism and the impact of Suvorexant on this process. 
The 5-day treatment paradigm of Suvorexant was sufficient to produce significant improvement of spatial recognition memory in female R6/1 mice. Our study confirms previous investigations reporting beneficial effects of sleep enhancement in cognitive performances in R6/2 mice [17-19]. In addition, our female mice outperformed male animals in cognitive, motor, and nest tests. Studies in HD mouse models have suggested sex-associated differences in the severity or the age of onset of the HD phenotype $[15,49,50]$, with females displaying less severe symptoms or a later development of the disease than males. These findings in mice are reminiscent of clinical observations in HD patients, where a later onset of motor HD alterations has been reported in women [64]. Indeed, a higher striatal susceptibility to oxidative stress as demonstrated in males [65], or estrogen-associated neuroprotection [66] also effective in many neuropathologies, may account for the sex differences observed in our study. In addition, a gender variation in the expression of pre pro-orexin during development and adulthood $[67,68]$ may also explain the differential sensibility to the antagonist of Orx treatment observed between our male and female R6/1 mice.

To conclude, our data suggest that the altered Orx activity pattern in HD mice plays a role in the generation of an HD-associated beta activity and in a disturbed sleep-wake behavior from 3 months of age. We also show that somewhat normal sleep may be restored following an acute pharmacological inhibition of Orx activity during the inactive period, and a 5-day treatment paradigm can induce significant physiological and behavioral changes in R6/1 mice. Further investigations are now required to better understand the alterations of the Orx system in detail in R6/1 and other HD mouse models and to identify the cellular mechanisms by which Orx antagonism is able to normalize the aberrant neural synchrony and sleep-wake cycle as well as to decrease the associated behavioral deficits.

Acknowledgments We thank Nicolas Mallet for his valuable help with preliminary experiment and Elodie Poinama and Nathalie Argenta for R6/ 1 mouse production, and Gill Courtand for help with image processing of immunofluorescence data.

Required Author Forms Disclosure forms provided by the authors are available with the online version of this article.

Funding This work was supported by Hereditary Disease Foundation to YHC.

\section{Compliance with Ethical Standards}

All experimental procedures were approved by the Institutional Animal Care and Use Committee, Comité d'Ethique pour l'Expérimentation Animale Bordeaux, and were in accordance with the European Communities Council Directive of 24 November 1986 (86/609/EEC).

\section{References}

1. The Huntington's disease collaborative research group. A novel gene containing a trinucleotide repeat that is expanded and unstable on Huntington's disease chromosomes/. Cell. 1993;72(6):971-83.

2. Raymond LA, André VM, Cepeda C, Gladding CM, Milnerwood AJ, Levine MS. Pathophysiology of Huntington's disease: timedependent alterations in synaptic and receptor function. Neuroscience. 2011;198:252-73.

3. Arnulf I, Nielsen J, Lohmann E, Schiefer J, Wild E, Jennum P, et al. Rapid eye movement sleep disturbances in Huntington disease. Arch Neurol. 2008;65(4):482-8.

4. Aziz NA, Anguelova GV, Marinus J, Lammers GJ, Roos RA. Sleep and circadian rhythm alterations correlate with depression and cognitive impairment in Huntington's disease. Parkinsonism Relat Disord. 2010;16(5):345-50.

5. Goodman AO, Rogers L, Pilsworth S, McAllister CJ, Shneerson $\mathrm{JM}$, Morton AJ, et al. Asymptomatic sleep abnormalities are a common early feature in patients with Huntington's disease. Curr Neurol Neurosci Rep. 2011;11(2):211-7.

6. Morton AJ. Circadian and sleep disorder in Huntington's disease. Exp Neurol. 2013;243:34-44.

7. Morton AJ, Wood NI, Hastings MH, Hurelbrink C, Barker RA, Maywood ES. Disintegration of the sleep-wake cycle and circadian timing in Huntington's disease. The Journal of neuroscience : the official journal of the Society for Neuroscience. 2005;25(1):157-63.

8. Kantor S, Szabo L, Varga J, Cuesta M, Morton AJ. Progressive sleep and electroencephalogram changes in mice carrying the Huntington's disease mutation. Brain : a journal of neurology. 2013;136(Pt 7):2147-58.

9. Fisher SP, Black SW, Schwartz MD, Wilk AJ, Chen TM, Lincoln WU, et al. Longitudinal analysis of the electroencephalogram and sleep phenotype in the R6/2 mouse model of Huntington's disease. Brain : a journal of neurology. 2013;136(Pt 7):2159-72.

10. Lebreton F, Cayzac S, Pietropaolo S, Jeantet Y, Cho YH. Sleep Physiology Alterations Precede Plethoric Phenotypic Changes in R6/1 Huntington's Disease Mice. PloS one. 2015;10(5):e0126972.

11. Kudo T, Schroeder A, Loh DH, Kuljis D, Jordan MC, Roos KP, et al. Dysfunctions in circadian behavior and physiology in mouse models of Huntington's disease. Exp Neurol. 2011;228(1):80-90.

12. Fisher SP, Schwartz MD, Wurts-Black S, Thomas AM, Chen TM, Miller MA, et al. Quantitative electroencephalographic analysis provides an early-stage indicator of disease onset and progression in the zQ175 knock-in mouse model of Huntington's disease. Sleep. 2016;39(2):379-91.

13. Loh DH, Kudo T, Truong D, Wu Y, Colwell CS. The Q175 mouse model of Huntington's disease shown gene dosage- and age-related decline in circadian rhythms of activity and sleep. PloS One. 2013;8(7):e69993.

14. Jeantet Y, Cayzac S, Cho YH. beta oscillation during slow wave sleep and rapid eye movement sleep in the electroencephalogram of a transgenic mouse model of Huntington's disease. PloS One. 2013;8(11):e79509.

15. Pietropaolo S, Delage P, Cayzac S, Crusio WE, Cho YH. Sexdependent changes in social behaviors in motor pre-symptomatic R6/1 mice. PloS one. 2011;6(5):e19965.

16. Du Z, Chazalon M, Bestaven E, Leste-Lasserre T, Baufreton J, Cazalets $\mathrm{JR}$, et al. Early GABAergic transmission defects in the external globus pallidus and rest/activity rhythm alteration in a mouse model of Huntington's disease. Neuroscience. 2016;329:363-79.

17. Pallier PN, Maywood ES, Zheng Z, Chesham JE, Inyushkin AN, Dyball R, et al. Pharmacological imposition of sleep slows cognitive decline and reverses dysregulation of circadian gene expression 
in a transgenic mouse model of Huntington's disease. The Journal of neuroscience : the official journal of the Society for Neuroscience. 2007;27(29):7869-78.

18. Pallier PN, Morton AJ. Management of sleep/wake cycles improves cognitive function in a transgenic mouse model of Huntington's disease. Brain research. 2009;1279:90-8.

19. Morton AJ, Hunt MJ, Hodges AK, Lewis PD, Redfern AJ, Dunnett $\mathrm{SB}$, et al. A combination drug therapy improves cognition and reverses gene expression changes in a mouse model of Huntington's disease. Eur J Neurosci. 2005;21(4):855-70.

20. Whittaker DS, Wand HB, Loh DH, Cachope R, Colwell CS. Possible use of a H3R antagonist for the management of nonmotor symptoms in the Q175 mouse model of Huntington's disease. Pharmacological research and perspective. 2017;5(5).

21. Cuesta M, Aungier J, Morton AJ. Behavioral therapy reverses circadian deficits in a transgenic mouse model of Huntington's disease. Neurobiology of disease. 2014;63:85-91.

22. Cuesta M, Aungier J, Morton AJ. The methamphetamine-sensitive circadian oscillator is dysfunctional in a transgenic mouse model of Huntington's disease. Neurobiology of disease. 2012;45(1):145-55.

23. Wang HB, Loh DH, Whittaker DS, Cutler T, Howland D, Colwell CS. Time-restricted feeding improves circadian dysfunction as well as motor symptoms in the Q175 mouse model of Huntington's disease. eNeuro. 2017;5(1):ENEURO.0431-17.2017.

24. Wang HB, Whittaker DS, Truong D, Mulji AK, Ghiani CA, Loh $\mathrm{DH}$, et al. Blue light therapy improves circadian dysfunction as well as motor symptoms in two mouse modeld of Huntington's disease. Neurobiology of sleep and circadian rhythms. 2017;2:39-52.

25. Hong SL, Cossyleon D, Hussain WA, Walker LJ, Barton SJ, Rebec GV. Dysfunctional behavioral modulation of corticostriatal communication in the R6/2 mouse model of Huntington's disease. PloS One. 2012;7(10):e47026.

26. Sakurai T, Amemiya, Ishii M, Matsuzaki I, Chemelli RM, Tanaka $\mathrm{H}$, et al. Orexins and orexin receptors: a family of hypothalamic neuropeptides and $\mathrm{G}$ protein-coupled receptors that regulate feeding behavior. Cell. 1998; 92(4), 573-585.

27. Sakurai T. (2007). The neural circuit of orexin (hypocretin): maintaining sleep and wakefulness. Nature Reviews Neuroscience, 8(3), 171.

28. Adamantidis AR, Zhang F, Aravanis AM, Deisseroth K, De Lecea L. Neural substrates of awakening probed with optogenetic control of hypocretin neurons. Nature. 2007; 450(7168), 420.

29. Hassani OK, Henny P, Lee MG, Jones BE. GABAergic neurons intermingled with orexin and $\mathrm{MCH}$ neurons in the lateral hypothalamus discharge maximally during sleep. European Journal of Neuroscience. 2010; 32(3), 448-457

30. Elias CF, Sita LV, Zambon BK, Oliveira ER, Vasconcelos LAP, Bittencourt JC. Melanin-concentrating hormone projections to areas involved in somatomotor responses. Journal of chemical neuroanatomy. 2008; 35(2), 188-201.

31. Varin C, Luppi PH, Fort P. Melanin-concentrating hormone-expressing neurons adjust slow-wave sleep dynamics to catalyze paradoxical (REM) sleep. Sleep. 2018; 41(6), zsy068.

32. Petersen A, Gil J, Maat-Schieman ML, Bjorkqvist M, Tanila H, Araujo IM, et al. Orexin loss in Huntington's disease. Hum Mol Genet. 2005;14(1):39-47.

33. Roos RA, Aziz NA. Hypocretin-1 and secondary signs in Huntington's disease. Parkinsonism Relat Disord. 2007;13 Suppl 3:S387-90.

34. Björkqvist M, Petersén A, Nielsen J, Ecker D, Mulder H, Hayden MR, et al. Cerebrospinal fluid levels of orexin-A are not a clinically useful biomarker for Huntington's disease. Clinical Genetics. 2006;70(1):78-90.

35. Meier A, Mollenhauer B, Cohrs S, Rodenbeck A, Jordan W, Meller $\mathrm{J}$, et al. Normal hypocretin-1 (orexin-A) levels in the cerebrospinal fluid of patients with Huntington's disease. Brain Research. 2005;1063(2):201-3.
36. Baumann CR, Hersberger M, Bassetti CL. Hypocretin-1 (orexin A) levels are normal in Huntington's disease. Journal of neurology. 2006;253(9):1232-3.

37. Williams RH, Morton AJ, Burdakov D. Paradoxical function of orexin/hypocretin circuits in a mouse model of Huntington's disease. Neurobiol Dis. 2011;42(3):438-45.

38. Sutton EL. Profile of suvorexant in the management of insomnia. Drug design, development and therapy. 2015; 9, 6035.

39. Mangiarini L, Sathasivam K, Seller M, Cozens B, Harper A, Hetherington $\mathrm{C}$, et al. Exon 1 of the $\mathrm{HD}$ gene with an expanded CAG repeat is sufficient to cause a progressive neurological phenotype in transgenic mice. Cell. 1996;87(3):493-506.

40. Jeantet Y, Cho YH. Design of a twin tetrode microdrive and headstage for hippocampal single unit recordings in behaving mice. Journal of Neuroscience Methods. 2003;129(2):129-34.

41. Gerbrandt LK, Lawrence JC, Eckardt MJ, Lloyd RL. Origin of the neocortically monitored theta rhythm in the curarized rat. Electroencephalography and clinical neurophysiology. 1978;45: 454-167.

42. Deacon RM. Assessing nest building in mice. Nature Protocol. 2006; 1(3):1117-9.

43. Hassani OK, Lee MG, Jones BE. Melanin-concentrating hormone neurons discharge in a reciprocal manner to orexin neurons across the sleep-wake cycle. Proceedings of the National Academy of Sciences of the United States of America. 2009;106(7):2418-22.

44. Broberger C, De Lecea L, Sutcliffe JG, Hökfelt T. Hypocretin/ orexin- and melanin-concentrating hormone-expressing cells form distinct populations in the rodent lateral hypothalamus: relationship to the neuropeptide $\mathrm{Y}$ and agouti gene-related protein systems. Journal of Comparative Neurolology. 1998;402(4):460-74.

45. Cvetkovic V, Brischoux F, Jacquemard C, Fellmann D, Griffond B, Risold PY. Characterization of subpopulations of neurons producing melanin-concentrating hormone in the rat ventral diencephalon. Journal of Neurochemistry. 2004;91(4):911-9.

46. Verret L, Goutagny R, Fort P, Cagnon L, Salvert D, Leger L, et al. A role of melanin-concentrating hormone producing neurons in the central regulation of paradoxical sleep. BMC neuroscience. 2003;4:19.

47. Naver B, Stub C, Moller M, Fenger K, Hansen AK, Hasholt L, et al. Molecular and behavioral analysis of the R6/1 Huntington's disease transgenic mouse. Neuroscience. 2003;122(4):1049-57.

48. Nithianantharajah J, Barkus C, Murphy M, Hannan AJ. Geneenvironment interactions modulating cognitive function and molecular correlates of synaptic plasticity in Huntington's disease transgenic mice. Neurobiol Dis. 2008;29(3):490-504.

49. Zielonka D, Marinus J, Roos RA, De Michele G, Di Donato S, Putter H, Marcinkowski J, Squitieri F, Bentivoglio AR, Landwehrmeyer GB. The influence of gender on phenotype and disease progression in patients with Huntington's disease. Parkinsonism Related Disorders. 2013;19(2):192-7.

50. Kuljis DA, Gad L, Loh DH, MacDowell Kaswan Z, Hitchcock ON, Ghiani CA, Colwell CS. Sex Differences in Circadian Dysfunction in the BACHD Mouse Model of Huntington's Disease. PLoS One. $2016 ; 11(2): \mathrm{e} 0147583$.

51. Kantor S, Varga J, Morton AJ. A single dose of hypnotic corrects sleep and EEG abnormalities in symptomatic Huntington's disease mice. Neuropharmacology. 2016;105:298-307.

52. Kantor S, Varga J, Kulkarni S, Morton AJ. Chronic Paroxetine treatment prevents the emergence of abnormal electroencephalogram oscillations in Huntington's disease mice. Neurotherapeutics. 2017;14(4): 1120-33.

53. Fox SV, Gotter AL, Tye SJ, Garson SL, Savitz AT, Uslaner JM, et al. Quantitative electroencephalography within sleep/wake states differentiates GABA A modulators eszopiclone and zolpidem from dual orexin receptor antagonists in rats. Neuropsychopharmacology, 2013; 38(12), 2401. 
54. Ma J, Svetnik V, Snyder E, Lines C, Roth T, Herring WJ. Electroencephalographic power spectral density profile of the orexin receptor antagonist suvorexant in patients with primary insomnia and healthy subjects. Sleep, 2014; 37(10), 1609-1619.

55. Morton AJ, Wood NI, Hastings MH, Hurelbrink C, Barker RA, Maywood ES. Disintegration of the sleep-wake cycle and circadian timing in Huntington's disease. The Journal of neuroscience : the official journal of the Society for Neuroscience. 2005;25(1):157-63.

56. Kuljis D, Kudo T, Tahara Y, Ghiani CA, Colwell CS. Pathophysiology in the suprachiasmatic nucleus in mouse models of Huntington's disease. J Neurosci Res. 2018;96(12):1862-1875.

57. Sakurai T, Mieda M. Connectomics of orexin-producing neurons: interface of systems of emotion, energy homeostatis and arousal. Trends in Pharmacological sciences. 2011;32(8):451-62.

58. Sakurai, T. (2014). The role of orexin in motivated behaviours. Nature Reviews Neuroscience, 15(11), 719.

59. Hara J, Beuckmann CT, Nambu T, Willie JT, Chemelli RM, Sinton $\mathrm{CM}$, et al. Genetic ablation of orexin neurons in mice results in narcolepsy, hypophagia, and obesity. Neuron. 2001 May;30(2): 345-54.

60. Ramanathan L, Siegel JM. Gender differences between hypocretin/ orexin knockout and wild type mice: age, body weight, body composition, metabolic markers, leptin and insulin resistance. $\mathrm{J}$ Neurochem. 2014 Dec;131(5):615-24.

61. Jöhren O, Neidert SJ, Kummer M, Dendorfer A, Dominiak P. Prepro-orexin and orexin receptor mRNAs are differentially expressed in peripheral tissues of male and female rats. Endocrinology. 2001 Aug;142(8):3324-31.

62. Funabashi T, Hagiwara H, Mogi K, Mitsushima D, Shinohara K, Kimura F. Sex differences in the responses of orexin neurons in the lateral hypothalamic area and feeding behavior to fasting. Neurosci Lett. 2009 Sep 29;463(1):31-4.

63. Pirnik Z, Bundzikova J, Mikkelsen JD, Zelezna B, Maletinska L, Kiss A. Fos expression in hypocretinergic neurons in C57B $1 / 6$ male and female mice after long-term consumption of high fat diet. Endocr Regul. 2008 Sep;42(4):137-46.

64. Roos RA, Vegter-van der Vlis M, Hermans J, Elshove HM, Moll AC, van de Kamp JJ, et al. Age at onset in Huntington's disease: effect of line of inheritance and patients's sex. Journal of medical genetics. 1991;28(8):515-9.

65. Rebec GV, Barton SJ, Ennis MD. Dysregulation of ascorbate release in the striatum of behaving mice expressing the Huntington's disease gene. Journal of neuroscience. 2002;22(2):RC202.

66. Simpkins, J. W., Yi, K. D., Yang, S. H., \& Dykens, J. A. (2010). Mitochondrial mechanisms of estrogen neuroprotection. Biochimica et Biophysica Acta (BBA)-General Subjects, 1800(10), 1113-1120.

67. Iwasa T, Matsuzaki T, Munkhzaya M, Tungalagsuvd A, Kuwahara A, Yasui T, et al. Developmental changes in the hypothalamic mRNA levels of prepro-orexin and orexin receptors and their sensitivity to fasting in male and female rats. Int $\mathrm{J}$ Dev Neurosci. 2015;46:51-4.

68. Johren O, Neidert SJ, Kummer M, Dominiak P. Sexually dimorphic expression of prepro-orexin mRNA in the rat hypothalamus. Peptides. 2002;23(6):1177-80

Publisher's Note Springer Nature remains neutral with regard to jurisdictional claims in published maps and institutional affiliations. 